\title{
Changes in meibomian gland morphology and ocular higher-order aberrations in eyes with chalazion
}

This article was published in the following Dove Press journal:

Clinical Ophthalmology

30 May 2017

Number of times this article has been viewed

\author{
Shima Fukuoka ${ }^{1-3}$ \\ Reiko Arita ${ }^{2-4}$ \\ Rika Shirakawa ${ }^{2,3}$ \\ Naoyuki Morishige 2,5 \\ 'Department of Ophthalmology, \\ Omiya Hamada Eye Clinic, ${ }^{2}$ Lid \\ and Meibomian Gland Working \\ Group (LIME), Saitama-shi, Saitama, \\ ${ }^{3}$ Department of Ophthalmology, \\ University of Tokyo School of \\ Medicine, Bunkyo-ku, Tokyo, \\ ${ }^{4}$ Department of Ophthalmology, \\ Itoh Clinic, Saitama-shi, Saitama, \\ ${ }^{5}$ Department of Ophthalmology, \\ Oshima Eye Hospital, Fukuoka-shi, \\ Fukuoka, Japan
}

Purpose: To examine the changes in meibomian gland morphology and ocular higher-order aberrations (HOAs) in eyes with chalazion and its excision.

Methods: Seven male patients with previous history of chalazion excision and seven control male subjects were enrolled. Changes in meibomian gland morphology (meiboscores, gland dropout, and shortening) were evaluated by using meibography equipped in a wavefront analyzer KR-1W and in a slit-lamp device BG-4M. Ocular HOAs were measured sequentially with KR-1W. The tear film breakup time (BUT) was measured.

Results: Both KR-1W and BG-4M visualized meibomian gland as clear similar images. The tear film BUT (mean $\pm \mathrm{SD}, 5.6 \pm 3.0$ vs $9.4 \pm 2.3$ seconds, $P=0.025$ ), the total meiboscore (median [interquartile range], $2(2-3)$ vs $0(0-1), P=0.007)$ as well as the meibomian gland dropout rate $(86 \%$ vs $14 \%, P=0.008)$ and shortening rate $(100 \%$ vs $29 \%, P=0.031)$ differed significantly between the patient and control groups. The first total ocular HOAs $(0.142 \pm 0.063$ vs $0.130 \pm 0.015, P=0.80$ ) were similar in both groups, whereas the stability index of the total HOAs over time $(0.0041 \pm 0.0048$ vs $-0.0012 \pm 0.0020, P=0.030)$ differed significantly between the patient and control groups.

Conclusions: Chalazion and its excision were associated with dropout and shortening rate of meibomian glands. The morphological changes of meibomian glands in chalazion may be associated to instability of the tear film, which was suggested by the tear film BUT and the stability of ocular HOAs.

Keywords: meibomian gland, meibography, wavefront analyzer, higher-order aberrations, chalazion excision

\section{Plain language summary}

Why was the study done? Chalazion is a small tumor of the eyelid arising from a blocked meibomian gland. Chalazion itself or chalazion excision may damage the associated meibomian glands. Changes in meibomian gland morphology after chalazion remain unclear, however. Chalazion excision was found to affect visual function soon after surgery. Changes in ocular higher-ocular aberrations (HOAs: small optical irregularities or imperfections of the eye which cannot be corrected by simple sphere and cylinder corrections) at several years after chalazion remain unclear. This study aimed to examine the effects of chalazion on meibomian glands and ocular HOAs.

What did the researchers do and find? Seven males after chalazion excision and seven control males were enrolled. We evaluated changes in meibomian gland morphology using two kinds of meibography. We measured ocular HOAs using the wavefront analyzer. We found that 1) dropout and shortening of meibomian glands were seen more often in patients than in controls; 2) the total HOAs showed an upward curve in patients, but remained constant in controls.
Correspondence: Reiko Arita
Department of Ophthalmology, Itoh Clinic, 626-II Minaminakano, Minuma-ku, Saitama-shi, Saitama 337-0042, Japan

$\mathrm{Tel}+8 \mathrm{I} 486865588$

Fax +8I 486868485

Email ritoh@za2.so-net.ne.jp
Clinical Ophthalmology 2017:1 I 1031-1038 (c) (1) (\$) $\odot 2017$ Fukuoka et al. This work is published and licensed by Dove Medical Press Limited. The full terms of this license are available at https://www.dovepress.com/terms.php

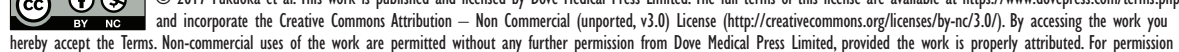
hereby accept the Terms. Non-commercial uses of the work are permitted without any further permisision from Dove Mediin
for commercial use of this work, please see paragraphs 4.2 and 5 of our Terms (https://www.dovepress. com/terms.php). 
What do these results mean? The morphological changes of meibomian glands in chalazion may be associated to instability of the tear film, which was suggested by the stability of ocular HOAs.

\section{Introduction}

Chalazion is a common eyelid disease. The International Workshop on Meibomian Gland Dysfunction refers to chalazion as a condition with localized involvement of meibomian glands. Chalazion may be causal of, or secondary to, meibomian gland dysfunction, or it may occur incidentally. ${ }^{1}$ Chalazion is a granulomatous inflammatory growth that forms within the upper or lower eyelid, or within both lids simultaneously, ${ }^{2}$ with chalazion excision being an effective surgery for lesions that do not heal. Chalazion itself or chalazion excision may damage the associated meibomian glands. Little is known of changes in meibomian gland morphology after chalazion formation and excision, however.

We have previously developed a noninvasive, patientfriendly meibographic system that is based on an infrared filter and an infrared charge-coupled device (CCD) camera attached to a slit-lamp device (BG-4M; Topcon, Tokyo, Japan) and which allows for a rapid and thorough examination for detection of morphological changes in meibomian glands throughout the eye. ${ }^{3}$ The wavefront analyzer (KR-1W; Topcon, Tokyo, Japan) combines aberrometry, topography, keratometry, pupillometry, and autorefraction. It is also equipped with an infrared (940 nm) observation system for the anterior eye to allow alignment. We have now modified this observation system to capture a still image of the meibomian glands.

Chalazion excision was found to reduce corneal astigmatism and irregularity at 1 month after surgery. ${ }^{4}$ Chalazion was also shown to increase higher-order aberrations (HOAs), as measured with a Hartmann-Shack aberrometer, and chalazion excision reduced HOAs at 2 months after surgery. ${ }^{5}$ However, changes in ocular HOAs at several years after chalazion excision have not been investigated.

We have now examined morphological changes of meibomian glands in patients with a history of chalazion excision both with the BG-4M system and with the KR-1W analyzer equipped with the newly modified program. We compared the results obtained with these two systems as well as examined the effects on chalazion of ocular HOAs.

\section{Methods}

\section{Subjects}

Male patients with a history of chalazion excision and agematched control male individuals (healthy volunteers) were enrolled in this study. Inclusion criteria for both groups included phakic, the pupil diameter of $>4 \mathrm{~mm}$ without mydriasis, and a best corrected visual acuity of 20/20 or better, which was sufficient to allow fixation on the target of the wavefront analyzer. Exclusion criteria for both groups included blepharitis, continuous eyedrop use, ophthalmic disorders (including superficial punctate keratopathy) other than refractive errors and mild cataracts, a history of previous ocular surgeries other than chalazion excision, and systemic or ocular diseases that might interfere with tear film production or function. The presented data were obtained from the left eye of each subject in the control group and from the eye with a history of chalazion in the patient group (if a patient had undergone surgery on both eyes, the left eye was selected for examination; if the left eye was excluded, data were obtained from the right eye). Measurements were performed in a room maintained at a temperature of $22^{\circ} \mathrm{C} \pm 3^{\circ} \mathrm{C}$ and a humidity of $40 \% \pm 4 \%$. The research protocol was approved by the institutional review board of the University of Tokyo School of Medicine, and written informed consent was obtained from each subject before examination. The study adhered to the tenets of the Declaration of Helsinki.

\section{Examinations}

The following tests were performed sequentially: measurement of ocular HOAs with KR-1W (by SF), a slit-lamp examination of lid margin abnormalities as well as of the cornea and conjunctiva before and after fluorescein staining, measurement of tear film breakup time (BUT) (by RA), assessment of tear production with Schirmer's test without topical anesthesia (by RA), noninvasive meibography with the modified KR-1W system (by SF) and with BG-4M (by $\mathrm{RS}$ ), and grading of meibum expression (by RS). The tests were conducted in this sequence in order to minimize the risk of one test interfering with the next one. ${ }^{6}$ Lid margin abnormalities were scored from 0 to 4 on the basis of the absence or presence of an irregular lid margin, vascular engorgement, plugging of meibomian gland orifices, or a shift in the mucocutaneous junction, with an absolute score of 0 or 1 for each of these four criteria (ie, the severity of each condition was not taken into consideration). The tear film BUT was measured three consecutive times after the instillation of fluorescein. The extent to which meibomian secretion (meibum) was induced in response to the application of digital pressure to the upper tarsus was evaluated semiquantitatively according to the following scale: ${ }^{7} 0$, clear meibum easily expressed; 1, cloudy meibum expressed with mild pressure; 2 , cloudy meibum expressed with moderate pressure; and 3, meibum not expressed, even with hard pressure. 


\section{Meibography}

We examined the morphology of meibomian glands both with the noncontact meibography system mounted on a slitlamp microscope ${ }^{3}$ (BG-4M) and with the wavefront analyzer (KR-1W) equipped with newly modified noninvasive meibography program, and we compared morphological changes of each gland duct and acinus after recording the photographs obtained with these two systems. Our new noninvasive meibography program for KR-1W comprises an infrared lightemitting diode (LED) (wavelength of $940 \pm 20 \mathrm{~nm}$ ) as a light source and an infrared CCD video camera (TCK237TPL; Texas Instruments, Dallas, TX, USA). When the eyelid is everted, an image of the meibomian glands, transilluminated from the conjunctival side by the infrared LED, is captured by the highly sensitive CCD camera. Less than 1 minute is required to observe the meibomian glands of both the upper and lower eyelids. Photographs of the meibomian glands can be digitally recorded.

The upper and lower eyelids were turned over, and the meibomian glands were observed with the two noninvasive infrared meibographic systems. Partial or complete loss of the meibomian glands was scored according to the following scale (meiboscore) for each eyelid: grade 0 , no loss of meibomian glands; grade 1 , the affected area was $<33 \%$ of the total area occupied by the meibomian glands; grade 2 , the affected area was between $33 \%$ and $66 \%$ of the total area occupied by the meibomian glands; grade 3, the affected area was $>66 \%$ of the total area occupied by the meibomian glands. ${ }^{3}$ Meiboscores for the upper and lower eyelids were summed for each eye. Quantitative analysis was performed to examine the similarity of the meibomian gland images by the two methods. The software for image analysis was used as previously described. ${ }^{8,9}$ Lid borders were automatically determined. The software evaluated the distribution of the luminance and, by enhancing the contrast and reducing image noise, the meibomian gland area was automatically discriminated. The software calculated the ratio of the total meibomian gland area relative to the total analysis area in each eyelid. To examine the similarity of the meibomian gland images obtained by the two methods, we compared the corresponding meiboscores and the ratios of meibomian gland area to total analysis area. The presence of dropout or shortening of the meibomian glands was also noted.

\section{Measurement of ocular HOAs}

Serial ocular HOAs were determined with the use of the program for dry eye of a Hartmann-Shack wavefront aberrometer (KR-1W). ${ }^{10-13}$ The details of this apparatus were described previously. ${ }^{14,15}$ Subjects were instructed to keep their eyes open for 10 seconds. The HOAs for a 4-mm pupil diameter were expanded into a set of orthogonal Zernike polynomials. The magnitudes of the coefficients of the Zernike polynomials are represented as the root mean square (RMS) and are used to show the wavefront aberrations. The RMS of the third-order Zernike coefficients (the square root of the sum of the squared coefficients of $\mathrm{C}_{3}{ }^{-3}$, $\mathrm{C}_{3}{ }^{-1}, \mathrm{C}_{3}{ }^{1}$, and $\mathrm{C}_{3}{ }^{3}$ ) was considered to represent a coma-like aberration, and the RMS of the fourth-order coefficients (the square root of the sum of the squared coefficients of $\mathrm{C}_{4}^{-4}, \mathrm{C}_{4}^{-2}$, $\mathrm{C}_{4}{ }^{0}, \mathrm{C}_{4}{ }^{2}$, and $\mathrm{C}_{4}^{4}$ ) was considered to denote a spherical-like aberration. The square root of the sum of the squared coefficients of $\mathrm{C}_{3}{ }^{-3}$ and $\mathrm{C}_{3}{ }^{3}$ was calculated as a trefoil aberration. Total HOAs were defined as the RMS of the magnitudes for the third- and fourth-order aberrations. The stability index (SI) of the total HOAs, ${ }^{10-13}$ defined as the slope of the linear regression line of the total ocular HOAs between blinks, was used to indicate the sequential change in HOAs over time. The first coma-like, spherical-like, and trefoil aberrations, the first total HOAs as well as the SI of the total HOAs over time were compared between the two subject groups.

\section{Statistical analysis}

Data are presented as median (interquartile range) for ordinal variables and mean values \pm standard deviations $(\mathrm{SD})$ for continuous variables. The differences in the average for age, lid margin abnormalities, BUT, Schirmer's test, meiboscores, meibum grades, coma-like, spherical-like, and trefoil aberrations, first total HOAs, as well as SI between the two groups of subjects were evaluated with the Mann-Whitney $U$-test. The frequencies of meibomian gland dropout and shortening were compared between the two groups with Fisher's exact test. The differences in the average for the ratios of meibomian gland area to total analysis area in each eyelid were evaluated with the Mann-Whitney $U$-test. The relation between these ratios using noncontact meibography equipped in a wavefront analyzer and in a slit-lamp microscope was examined with Spearman's correlation coefficient. The relation between the time and the total HOAs in the representative patient with a history of chalazion was analyzed with Spearman's correlation coefficient. A $P$-value of $<0.05$ was considered statistically significant.

\section{Results}

\section{Demographics}

Seven male patients with a history of chalazion (age, mean $\pm \mathrm{SD}, 44.3 \pm 11.2$ years, range, $29-56$ years) and seven age-matched control male individuals (healthy volunteers; age, mean $\pm \mathrm{SD}, 38.0 \pm 8.2$ years, range, 29-53 years) were 
Table I Tear film parameters for patients with a history of chalazion excision and control subjects

\begin{tabular}{llll}
\hline Parameters & $\begin{array}{l}\text { Patients with a history of } \\
\text { chalazion excision }(\mathbf{n}=\mathbf{7})\end{array}$ & Controls $(\mathbf{n}=\mathbf{7})$ & P-value \\
\hline Lid margin abnormality score $(0-4)$ & $2(0-3)$ & $0(0-I)$ & 0.092 \\
Meibum grade $(0-3)$ & $I(0-2)$ & $I(0-I)$ & 0.54 \\
Schirmer's test value $(\mathrm{mm})$ & $15.7 \pm I I .5$ & $26.4 \pm 8.1$ & 0.070 \\
BUT with fluorescein $($ seconds) & $5.6 \pm 3.0$ & $9.4 \pm 2.3$ & $0.025^{*}$ \\
Coma-like aberrations $(\mu \mathrm{m})$ & $0.114 \pm 0.042$ & $0.120 \pm 0.019$ & $0.6 I$ \\
Spherical-like aberrations $(\mu \mathrm{m})$ & $0.085 \pm 0.053$ & $0.047 \pm 0.015$ & 0.20 \\
Trefoil aberrations $(\mu \mathrm{m})$ & $0.129 \pm 0.19 \mid$ & $0.063 \pm 0.020$ & 0.90 \\
First total ocular HOAs & $0.142 \pm 0.063$ & $0.130 \pm 0.015$ & 0.80 \\
Sl of total HOAs & $0.004 I \pm 0.0048$ & $-0.0012 \pm 0.0020$ & $0.030^{*}$ \\
\hline
\end{tabular}

Notes: Data are median (interquartile range) for ordinal variables and mean values \pm standard deviations for continuous variables. $P$-values were determined with the Mann-Whitney $U$-test; $* P<0.05$.

Abbreviations: BUT, breakup time; HOAs, higher-order aberrations; SI, stability index.

enrolled in the study. No significant difference in age was apparent between the two groups of eyes $(P=0.34)$. Patients underwent the chalazion excision $20.6 \pm 15.9$ (range, 3-50) years before the examination.

\section{Tear film parameters}

Tear film parameters for the patients with a history of chalazion excision and control individuals are shown in Table 1. Tear film BUT (mean \pm SD, $5.6 \pm 3.0$ vs $9.4 \pm 2.3$ seconds, $P=0.025)$ differed significantly between the patient and control groups. There was no significant difference in the lid margin abnormality score, the meibum grade, or Schirmer's test value between the two groups of subjects.

\section{Meibography}

Normal meibomian glands were detected as long, straight, and narrow grape-like clusters of numerous small hyperilluminated spots, similarly corresponding to individual acini. Comparison of the ratios of meibomian gland area to total analysis area obtained with the noncontact meibography system mounted on a slit-lamp microscope and with the modified version of the KR-1W wavefront analyzer revealed a similar ability to detect morphological changes in meibomian glands (Table 2). Occasionally, the pictures for the new noninvasive meibography program attached to the wavefront analyzer did not be as sharp as those for the noncontact meibography equipped with a slit-lamp. Focal blurry part of the pictures were detected in 2 of the 14 eyes $(14 \%)$ in the upper and lower eyelids, respectively, but it was able to score the grades for each eyelid and detect the presence of gland dropout or shortening of the meibomian glands. Both systems thus similarly detected meibomian gland dropout and shortening in patients with a history of chalazion (Figure 1). For all examined eyelids of both the control and patient groups, the meiboscore obtained with the modified KR-1W system was the same as that obtained with the noncontact meibography system attached to a slitlamp. Meibomian gland dropout was observed in 6 of 7 eyes $(86 \%)$ in the patient group and in 1 of 7 eyes $(14 \%)$ in the control group $(P=0.008)$. Meibomian gland shortening was observed in 7 of 7 eyes (100\%) in the patient group and in 2 of 7 eyes (29\%) in the control group ( $P=0.031$; Table 3$)$. Focal meibomian gland dropout or shortening was detected in 4 of 7 eyes (57\%) in the patient group, whereas 4 of the 7 eyes (57\%) in this group manifested multiple or diffuse morphological changes of meibomian glands.

Table 2 Ratio of meibomian gland area to total analysis area using noncontact meibography equipped in a wavefront analyzer and in a slit-lamp microscope

\begin{tabular}{|c|c|c|c|c|c|}
\hline \multirow[t]{2}{*}{ Eyelid } & \multicolumn{2}{|c|}{$\begin{array}{l}\text { Ratio of meibomian gland area to total } \\
\text { analysis area (\%) }\end{array}$} & \multirow{2}{*}{$\begin{array}{l}\text { Mann-Whitney, } \\
U \text {-test } \\
P \text {-value }\end{array}$} & \multicolumn{2}{|c|}{$\begin{array}{l}\text { Spearman's } \\
\text { correlation coefficient }\end{array}$} \\
\hline & $\begin{array}{l}\text { Wavefront analyzer } \\
(\mathrm{n}=12)(\text { mean } \pm \text { SD })\end{array}$ & $\begin{array}{l}\text { Slit-lamp microscope } \\
(\mathrm{n}=12)(\text { mean } \pm \text { SD })\end{array}$ & & $\rho$ & $P$-value \\
\hline Upper eyelid & $45.2 \pm 9.5$ & $48.7 \pm 7.2$ & 0.064 & 0.636 & $0.026^{*}$ \\
\hline Lower eyelid & $49.5 \pm 11.1$ & $54.1 \pm 10.6$ & 0.15 & 0.615 & $0.033^{*}$ \\
\hline
\end{tabular}

Notes: $P$-values for two averages were evaluated with the Mann-Whitney $U$-test. Spearman's correlation coefficient $(\rho)$ and $P$-values were determined for the relation between the ratios of meibomian gland area to total analysis area in each eyelid; $* P<0.05$.

Abbreviation: SD, standard deviation. 

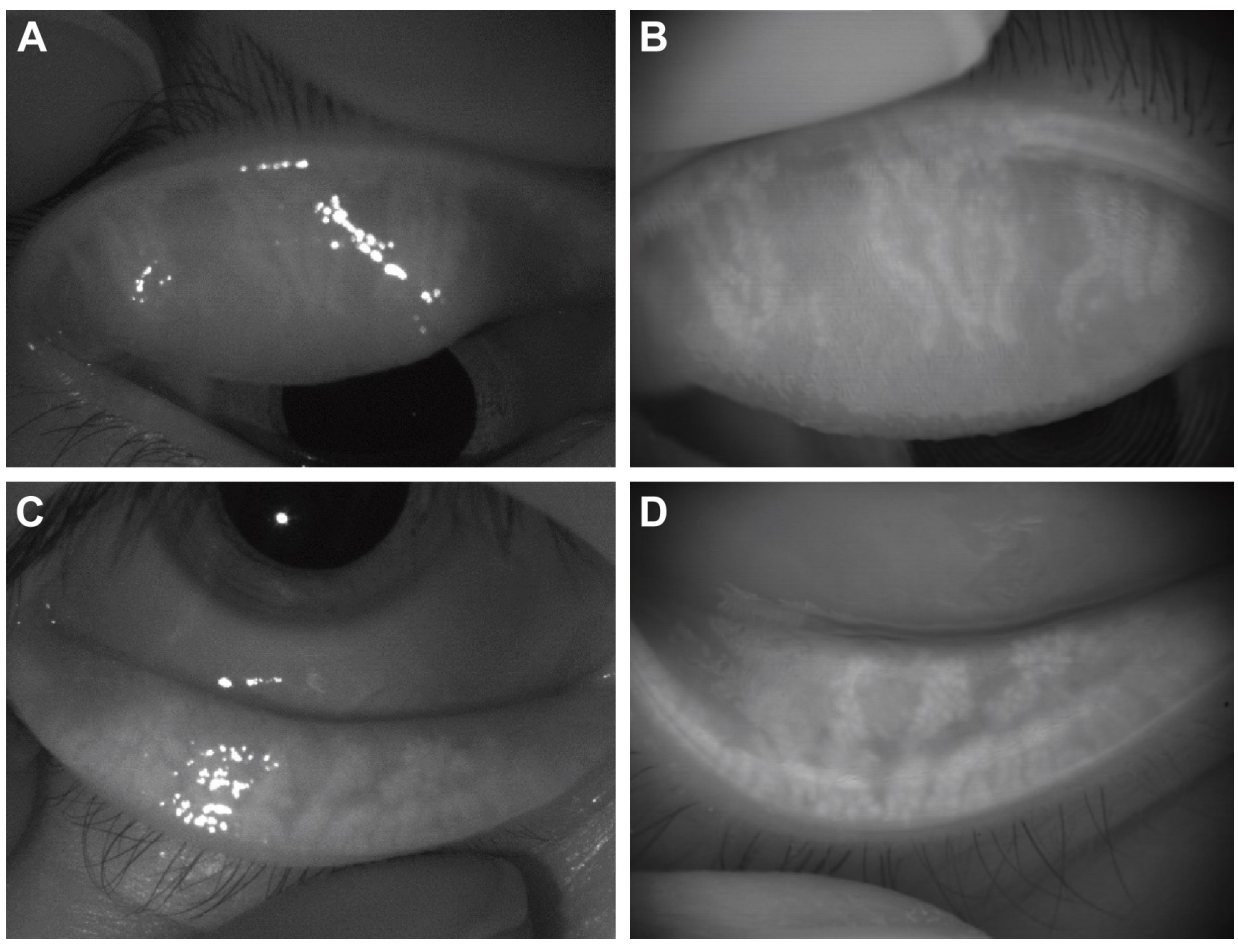

Figure I Representative images of an eye with a history of chalazion.

Notes: Images of meibomian gland structures in the upper (A, B) and lower (C, D) right eyelids of a 29-year-old man with a history of chalazion in the upper right eyelid were obtained with the noncontact meibography system attached to a slit-lamp (BG-4M) (A, C) and with the wavefront analyzer (KR-IW) equipped with the newly developed meibography program (B, D). Meibomian gland dropout was apparent in the upper eyelid, and meibomian gland shortening was detected in both the upper and lower eyelids. The quality of the images obtained with the two systems was similar.

\section{Measurement of ocular HOAs}

The wavefront color-coded maps of ocular HOAs obtained between blinks for representative individuals in the patient and control groups are shown together with the changes in the simulated retinal images of a Landolt ring (optotype with $\log$ MAR value of +0.7 ) in Figure 2. In healthy eyes, the sequential total HOAs remained constant at each blink (Figures 2A and 3A); no marked deterioration was thus apparent in consecutive wavefront maps and simulated retinal images. In contrast, the total HOAs tended to show an upward

Table 3 Meiboscores (median [interquartile range]) and frequency of meibomian gland dropout or shortening in patients with a history of chalazion excision and control subjects

\begin{tabular}{llll}
\hline Parameters & $\begin{array}{l}\text { Patients with } \\
\text { a history of } \\
\text { chalazion (n=7) }\end{array}$ & $\begin{array}{l}\text { Controls } \\
(\mathbf{n}=\mathbf{7})\end{array}$ & \\
\hline Meiboscore (upper eyelid) (0-3) & $2(1-2)$ & $0(0-1)$ & $0.008^{*}$ \\
Meiboscore (lower eyelid) (0-3) & $1(0-1)$ & $0(0-0)$ & $0.042^{*}$ \\
Meiboscore (total) (0-6) & $2(2-3)$ & $0(0-1)$ & $0.007^{*}$ \\
Dropout & $6(86 \%)$ & $\mathrm{I}(14 \%)$ & $0.008^{*}$ \\
Shortening & $7(100 \%)$ & $2(29 \%)$ & $0.03 I^{*}$ \\
\hline
\end{tabular}

Notes: $P$-values were determined with the Mann-Whitney U-test or Fisher's exact test; $* P<0.05$. curve between blinks in patients with a history of chalazion (Figures 2B and 3B). Table 1 shows the mean coma-like, spherical-like, and trefoil aberrations, first total ocular HOAs and the SI calculated from the data obtained from both patients and control subjects. The coma-like, spherical-like, and trefoil aberrations, first total ocular HOAs were similar $(P=0.61$, $0.20,0.90$, and 0.80 , respectively) for the two groups, whereas the SI of the total HOAs over time was significantly $(P=0.030)$ greater for patients than for controls.

\section{Discussion}

In this study, we obtained clear images of the meibomian glands of all subjects using either the noninvasive meibography system attached to a slit-lamp ${ }^{3}$ (BG-4M) or the newly developed noninvasive meibography system based on the KR-1W wavefront analyzer. Both systems showed similar abilities to detect morphological changes of meibomian glands (Table 2). Noninvasive meibography has previously been demonstrated to be highly useful for observing such morphological changes. ${ }^{3,8,9,16-22}$ In this study, gland dropout and shortening were observed in patients with a history of chalazion, consistent with previous findings obtained with the Keratograph 4 (OCULUS, Wetzlar, Germany) equipped 


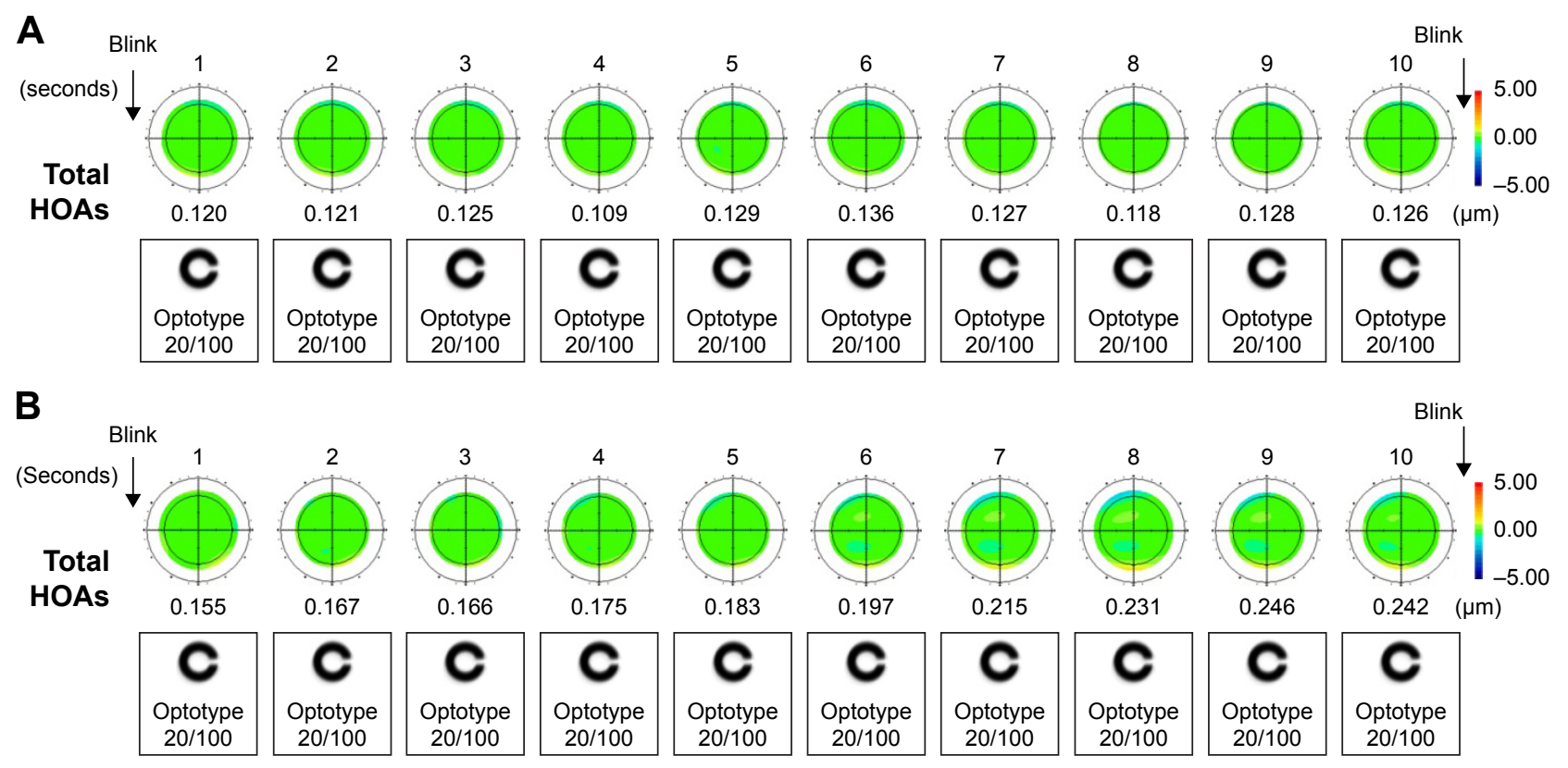

Figure 2 Sequential wavefront color-coded maps of total ocular HOAs in a control subject and a representative patient with a history of chalazion.

Notes: (A) A 43-year-old male control subject. The first total ocular HOA was 0.120 . (B) A 4I-year-old man with history of chalazia three times in the left eye. The first total ocular HOA was 0.155 . The maps are shown for I-second intervals together with changes in the simulated retinal images of a Landolt ring (optotype with logMAR value of +0.7$)$.

Abbreviation: HOA, higher-order aberrations.

with an infrared illumination system showing that the area of recurrent chalazion exhibited partial or complete meibomian gland loss and that active chalazia as well as the site of chalazion incision and drainage manifested meibomian gland dropout. ${ }^{23}$ The changes in meibomian gland morphology were apparent many years after chalazion development and its excision in this study.
The meibomian glands are specialized holocrine glands that secrete the lipid layer of the tear film, which helps prevent tear film evaporation. Localized involvement of meibomian glands in conditions such as chalazion has been thought not to result in tear film abnormalities. ${ }^{24} \mathrm{~A}$ recent study showed that the area of meibomian gland loss correlates inversely with lipid layer thickness, and that lipid layer thickness correlates
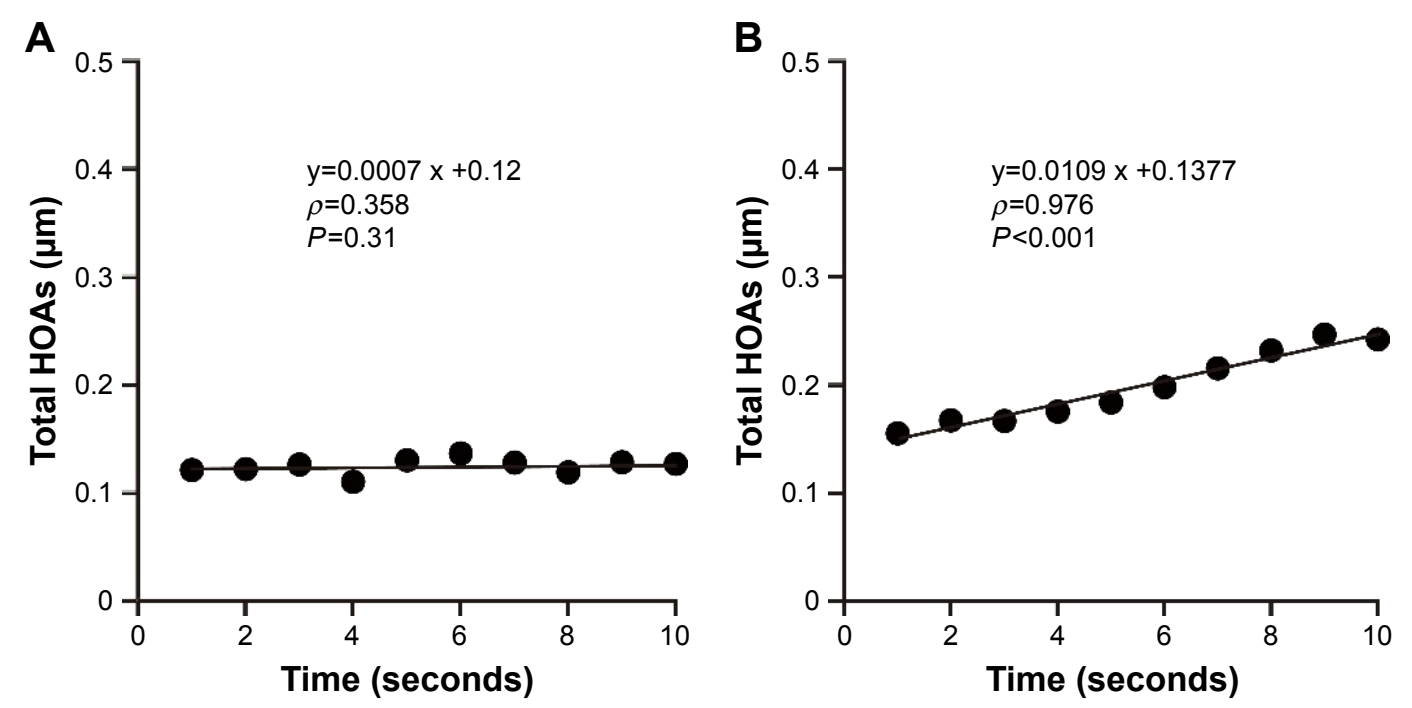

Figure 3 Sequential changes in total HOAs over 10 seconds after blinking and the corresponding linear regression curves for a control subject and a representative patient with a history of chalazion.

Notes: Spearman's correlation coefficient $(\rho)$ and corresponding $P$-values are shown. (A) A 43-year-old control male subject. The SI of the total HOAs over time was 0.0007. (B) A 4I-year-old man with history of chalazia three times in the left eye. The SI of the total HOAs over time was 0.0I09.

Abbreviations: HOAs, higher-order aberrations; SI, stability index. 
positively with tear film BUT, however. ${ }^{25}$ In this study, the tear film BUT was significantly shorter and meiboscores were significantly higher in the patients with a history of chalazion excision than that in control subjects, whereas the lid margin abnormality score, Schirmer's test values, or meibum grade did not differ significantly between the two groups. We also found that the coma-like, spherical-like, and trefoil aberrations, as well as the first total ocular HOAs were similar in the two groups of subjects. Whereas the sequential total HOAs remained constant over time in our control subjects, they tended to show an upward curve in the patients with a history of chalazion excision. The SI for the total HOAs over time was thus significantly greater in the patient group than in the control group. Given that BUT is affected by not only the lipid layer but also the mucin and aqueous layers of the tear film, tear film stability is likely influenced by different tear film components, such as meibomian lipids, ocular mucins, and proteins. ${ }^{26}$ The aqueous component of tear film was not deficient in the patients in our study. Moreover, sequential changes in total HOAs in subjects with a short tear film BUT were previously found to show a sawtooth pattern with a marked upward curve that increased after blinking. ${ }^{12}$ These various observations suggest that morphological changes of meibomian glands may lead to instability of the tear film.

Some of the patients with a history of chalazion in this study had experienced recurrent episodes of chalazion or meibomian gland concretion. Furthermore, some of the lids of the control subjects manifested morphological changes of meibomian glands. Four of 7 eyes (57\%) in the patient group showed multiple or diffuse morphological changes of meibomian glands. Given that the International Workshop on Meibomian Gland Dysfunction considers that chalazion may be associated with meibomian gland dysfunction, ${ }^{27}$ patients in this study essentially may have had this condition.

Occasionally, the pictures for the new noninvasive meibography program equipped to the wavefront analyzer (KR-1W) were not as clear as those for the noncontact meibography equipped with a slit-lamp. We speculated that the light source of each imaging tool caused difference of the meibomian gland images. The noncontact meibography equipped with a slit-lamp uses a point light source; however, KR-1 W wavefront analyzer equipped noninvasive meibography program was applied with a ring illumination for videokeratography. Specular reflection of the ring illumination could stripe and blur the part of the meibography pictures and make it difficult to distinguish meibomian gland area from nonmeibomian gland area. In addition to that, the difference in resolution and sensitivity of the equipped digital camera between the two instruments may be one of the cause of the different images of meibography. Overall, the meibomian gland images obtained by the wavefront analyzer (KR-1W) equipped with newly modified noninvasive meibography program were sufficient for analyzing meibomian gland morphology.

Our study has some limitations. Detailed information on the precise location of chalazion and the surgical procedure were not available for the patients with a history of chalazion and its excision, because of the retrospective design of the study. We thus were not able to observe the morphology of meibomian glands before chalazion formation or before its excision. Furthermore, the observed increase in the frequency of meibomian gland dropout or shortening, in the study, patients might be the result of chalazion itself or of the invasive surgery. Prospective clinical studies will be required to confirm the effects of chalazion itself and its excision on the morphology of meibomian glands and ocular HOAs.

\section{Conclusion}

Chalazion itself and chalazion excision changed the morphology of meibomian glands and thereby might affect meibomian gland function as well as visual function.

\section{Acknowledgments}

The authors thank Mr Hideaki Tokoro, Mr Tsuyoshi Haraguchi, and Mr Jun Suehiro of Topcon (Itabashi, Tokyo, Japan) for their help in constructing this wavefront analyzer equipped newly modified noninvasive meibography program. The authors also would like to thank Dr Shiro Amano of Inoue Eye Hospital (Chiyoda, Tokyo, Japan) for his advice.

\section{Disclosure}

Dr Arita holds a patent on the noncontact meibography system used in the study (JP Patent Registration No 5281846, US Patent Publication No 2011-0273550A1, EP Patent Publication No 2189108A1). Drs Fukuoka, Shirakawa, and Morishige report no conflicts of interest in this work.

\section{References}

1. Nelson JD, Shimazaki J, Benitez-del-Castillo JM, et al. The international workshop on meibomian gland dysfunction: report of the definition and classification subcommittee. Invest Ophthalmol Vis Sci. 2011;52(4): 1930-1937.

2. Mueller JB, McStay CM. Ocular infection and inflammation. Emerg Med Clin North Am. 2008;26(1):57-72, vi.

3. Arita R, Itoh K, Inoue K, Amano S. Noncontact infrared meibography to document age-related changes of the meibomian glands in a normal population. Ophthalmology. 2008;115(5):911-915. 
4. Bagheri A, Hasani HR, Karimian F, Abrishami M, Yazdani S. Effect of chalazion excision on refractive error and corneal topography. Eur J Ophthalmol. 2009;19(4):521-526.

5. Sabermoghaddam AA, Zarei-Ghanavati S, Abrishami M. Effects of chalazion excision on ocular aberrations. Cornea. 2013;32(6):757-760.

6. Foulks GN, Bron AJ. Meibomian gland dysfunction: a clinical scheme for description, diagnosis, classification, and grading. Ocul Surf. 2003; 1(3):107-126.

7. Shimazaki J, Goto E, Ono M, Shimmura S, Tsubota K. Meibomian gland dysfunction in patients with Sjögren syndrome. Ophthalmology. 1998;105(8):1485-1488.

8. Arita R, Itoh K, Maeda S, Maeda K, Amano S. A newly developed noninvasive and mobile pen-shaped meibography system. Cornea. 2013; 32(3):242-247.

9. Arita R, Suehiro J, Haraguchi T, Shirakawa R, Tokoro H, Amano S. Objective image analysis of the meibomian gland area. Br J Ophthalmol. 2014;98(6):746-755.

10. Koh S, Maeda N, Hirohara Y, et al. Serial measurements of higherorder aberrations after blinking in normal subjects. Invest Ophthalmol Vis Sci. 2006;47(8):3318-3324.

11. Koh S, Maeda N, Hamano T, et al. Effect of internal lubricating agents of disposable soft contact lenses on higher-order aberrations after blinking. Eye Contact Lens. 2008;34(2):100-105.

12. Koh S, Maeda N, Hori Y, et al. Effects of suppression of blinking on quality of vision in borderline cases of evaporative dry eye. Cornea. 2008;27(3):275-278.

13. Koh S, Maeda N, Hirohara Y, et al. Serial measurements of higher-order aberrations after blinking in patients with dry eye. Invest Ophthalmol Vis Sci. 2008;49(1):133-138.

14. Piñero DP, Juan JT, Alió JL. Intrasubject repeatability of internal aberrometry obtained with a new integrated aberrometer. J Refract Surg. 2011;27(7):509-517.

15. López-Miguel A, Martínez-Almeida L, González-García MJ, Coco-Martín MB, Sobrado-Calvo P, Maldonado MJ. Precision of higher-order aberration measurements with a new Placido-disk topographer and Hartmann-Shack wavefront sensor. J Cataract Refract Surg. 2013; 39(2):242-249.

16. Arita $\mathrm{R}$, Itoh $\mathrm{K}$, Inoue $\mathrm{K}$, Kuchiba $\mathrm{A}$, Yamaguchi $\mathrm{T}$, Amano S. Contact lens wear is associated with decrease of meibomian glands. Ophthalmology. 2009;116(3):379-384.
17. Arita R, Itoh $\mathrm{K}$, Maeda $\mathrm{S}$, et al. Proposed diagnostic criteria for obstructive meibomian gland dysfunction. Ophthalmology. 2009; 116(11):2058-2063.e2051.

18. Arita R, Itoh K, Maeda S, Maeda K, Tomidokoro A, Amano S. Efficacy of diagnostic criteria for the differential diagnosis between obstructive meibomian gland dysfunction and aqueous deficiency dry eye. Jpn J Ophthalmol. 2010;54(5):387-391.

19. Arita R, Itoh $\mathrm{K}$, Maeda $\mathrm{S}$, et al. Meibomian gland duct distortion in patients with perennial allergic conjunctivitis. Cornea. 2010;29(8): 858-860.

20. Arita R, Itoh $\mathrm{K}$, Maeda $\mathrm{S}$, et al. Proposed diagnostic criteria for seborrheic meibomian gland dysfunction. Cornea. 2010;29(9):980-984.

21. Arita R, Itoh $\mathrm{K}$, Maeda S, Maeda K, Tomidokoro A, Amano S. Association of contact lens-related allergic conjunctivitis with changes in the morphology of meibomian glands. Jpn JOphthalmol. 2012;56(1): 14-19.

22. Shirakawa R, Arita R, Amano S. Meibomian gland morphology in Japanese infants, children, and adults observed using a mobile penshaped infrared meibography device. Am J Ophthalmol. 2013;155(6): 1099.e1-1103.e1.

23. Srinivasan S, Menzies KL, Sorbara L, Jones LW. Imaging meibomian glands on a patient with chalazia in the upper and lower lids: a case report. Cont Lens Anterior Eye. 2013;36(4):199-203.

24. Nichols KK, Foulks GN, Bron AJ, et al. The international workshop on meibomian gland dysfunction: executive summary. Invest Ophthalmol Vis Sci. 2011;52(4):1922-1929.

25. Eom Y, Lee JS, Kang SY, Kim HM, Song JS. Correlation between quantitative measurements of tear film lipid layer thickness and meibomian gland loss in patients with obstructive meibomian gland dysfunction and normal controls. Am J Ophthalmol. 2013;155(6):1104-1110.e2.

26. Sweeney DF, Millar TJ, Raju SR. Tear film stability: a review. Exp Eye Res. 2013;117:28-38.

27. Geerling G, Tauber J, Baudouin C, et al. The international workshop on meibomian gland dysfunction: report of the subcommittee on management and treatment of meibomian gland dysfunction. Invest Ophthalmol Vis Sci. 2011;52(4):2050-2064.
Clinical Ophthalmology

\section{Publish your work in this journal}

Clinical Ophthalmology is an international, peer-reviewed journal covering all subspecialties within ophthalmology. Key topics include: Optometry; Visual science; Pharmacology and drug therapy in eye diseases; Basic Sciences; Primary and Secondary eye care; Patient Safety and Quality of Care Improvements. This journal is indexed on Submit your manuscript here: http://www.dovepress.com/clinical-ophthalmology-journal

\section{Dovepress}

PubMed Central and CAS, and is the official journal of The Society of Clinical Ophthalmology (SCO). The manuscript management system is completely online and includes a very quick and fair peer-review system, which is all easy to use. Visit http://www.dovepress.com/ testimonials.php to read real quotes from published authors. 